\title{
Time-domain Assessment of Data Transmission Errors in Systems with Multiple DC/DC Converters
}

\author{
$1^{\text {st }}$ Karol Niewiadomski \\ GGIEMR \\ University of Nottingham \\ Nottingham, United Kingdom \\ karol.niewiadomski@nottingham.ac.uk \\ $4^{\text {th }}$ Jacek Bojarski \\ Dept. of Math. Statistics and Econometrics, \\ University of Zielona Góra \\ Zielona Góra, Poland \\ j.bojarski@wmie.uz.zgora.pl
}

\author{
$2^{\text {nd }}$ Piotr Leżyński \\ Institute of Electrical Engineering \\ University of Zielona Góra \\ Zielona Góra, Poland \\ p.lezynski@iee.uz.zgora.pl \\ $5^{\text {th }}$ Mark Sumner \\ PEMC \\ University of Nottingham \\ Nottingham, United Kingdom \\ mark.sumner@nottingham.ac.uk
}

\author{
$3^{\text {rd }}$ Robert Smoleński \\ Institute of Electrical Engineering \\ University of Zielona Góra \\ Zielona Góra, Poland \\ r.smolenski@iee.uz.zgora.pl
}

\author{
$6^{\text {th }}$ David W. P. Thomas \\ GGIEMR \\ University of Nottingham \\ Nottingham, United Kingdom \\ dave.thomas@nottingham.ac.uk
}

\begin{abstract}
In this paper a statistical approach to quantify time-domain impact of common mode (CM) voltage induced on a communication system by the operation of several DC/DC converters is presented. The mathematical formulation of a problem of conducted electromagnetic interference (EMI) affecting the communication link is derived, where inductively coupled voltages are expressed in terms of damped harmonic oscillations. Monte Carlo (MC) simulations for assessing the density of times between interferences are conducted and preliminary results are shown. These results constitute a basis for creation of a model expressing the probability of communication errors encountered in systems with multiple sources of emissions.
\end{abstract}

Index Terms-Conducted EMI, EMC, statistical modeling, Monte Carlo simulations, DC/DC converters

\section{INTRODUCTION}

Power Converters (PC) are used in Smart Grid systems, acting as interfaces between the grid and energy sources. [1]. However, due to the switching action of the transistors a considerable amount of Electromagnetic Interference (EMI) is produced. EMI currents and voltages, induced by operation of power converters, may cause Electromagnetic Compatibility (EMC) issues [2] [3] [4]. According to European EMC 2014/30/EU Directive electromagnetic compatibility means "the ability of equipment to function satisfactorily in its electromagnetic environment without introducing intolerable electromagnetic disturbances to other equipment in that environment". In order to make an apparatus available in the market, it has to pass standardized tests, which focus on the frequency content of the Conducted Emissions (CE) generated by the device under test. Yet, recent studies have shown that fulfillment of standards defined in the frequency domain does not imply that the apparatus has electromagnetic compatibility within EMC definition [5] [6]. They show the issues arising from time variability of currents and voltages present in the

This project research is funded by the European Union's Horizon 2020 research and innovation programme under the Marie Sklodowska-Curie grant agreement No 812391 . considered systems. A particular issue related to the timevariability of interference is its influence on the control signal, where signal values at specific time instants may bring about unwanted side effects [7]. A time-domain (T-D) analysis was therefore postulated [5] [8] and further work is needed to develop rigorous and standardized approaches for timedomain testing. Moreover, harmonised standards do not offer a precise description of evaluating EMC in systems with many interference sources [9]. As has already been presented, systems consisting of multiple emission sources can produce significantly more EMI than those consisting of single emission source [6] [9] [10]. In such systems, currents are spread over in differential mode (DM) and common mode (CM) circuits [11] [12]. Therefore, a natural question arises: is there a way to predict the behaviour of the system, where multiple emission sources are present, i.e. its impact on a communication channel? Answer to this question would be valuable from both cognitive and practical point of view. The aim of this research is to describe the impact of several emission sources on a communication system. The results should give ways to model the aggregated interferences, which could be used for the development of standards that include time-domain effects. For the simplicity of reasoning, a deterministic Pulse Width Modulation (PWM) operated DC/DC converter was chosen as an emission source. A mathematical formulation of the problem is suggested and preliminary results of the simulation are presented.

\section{CM VOLTAGE GENERATED B Y GROUP OF DC/DC CONVERTERS}

Fig. 1 shows a half-bridge DC/DC converter used to obtain the preliminary measurements. Fig. 2 shows the schematic diagram of the experimental system, where two DC/DC converters supply the resistive loads. The differential mode current flows through the load (the internal parts of the circuitry). For security reasons, we often need to ground the conductive 


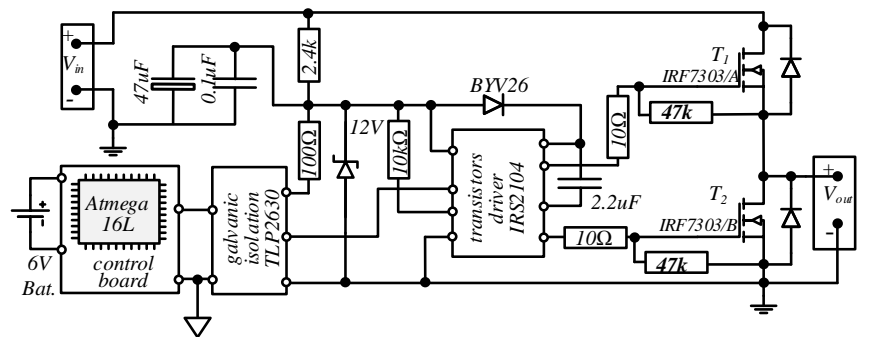

Fig. 1. Schematic of a half-bridge dc/dc converter with auxiliary elements.

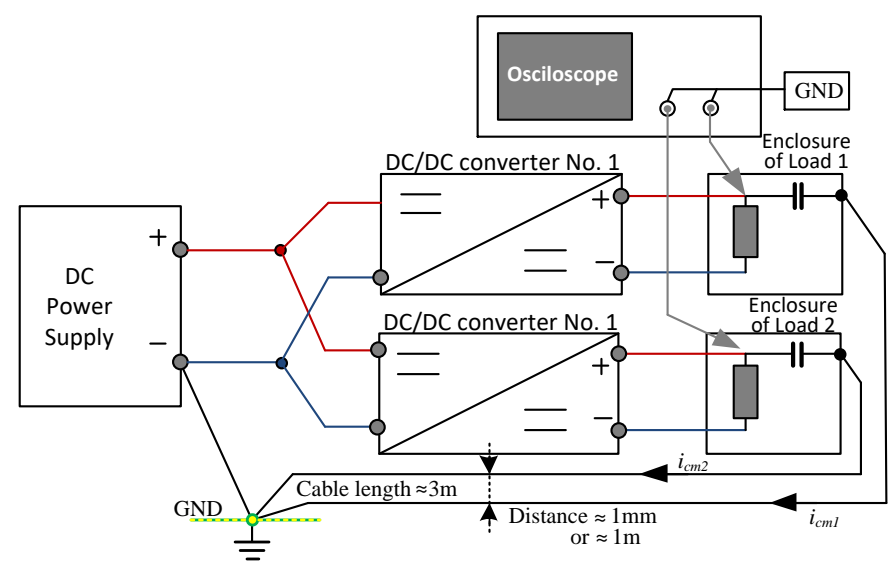

Fig. 2. Schematic of a system with power supply, dc/dc converter and a load in grounded enclosure.

enclosure of the load. In such case, parasitic capacitance may exist between the load and its enclosure, closing a circuit for common mode $(\mathrm{CM})$ current $i_{c m}$. In the presented experimental system, we simulated these capacitances by placing $5 \mathrm{nF}$ capacitors between the positive load clamp and grounded cables.

Excitation of common mode current leads to voltage drops on the series circuitry elements. Fig. 3 shows the output to ground voltage waveforms, measured according to Fig. 2. As one may see, the voltage has the shape of damped high frequency oscillations. The frequency of oscillation mode depends on the CM circuit's RLC parameters and may be identified in pursuance with [3]. When separate control systems control the converters, the output signal waveforms are not synchronized, as presented on Fig. 3 b). On Fig. 4, the damped oscillation voltages are enlarged. Panel a) presents a situation where grounding cables run in parallel preserving a distance of $1 \mathrm{~m}$ between each other. Moreover, Fig. $4 \mathrm{~b}$ ) shows the effect of coupling between CM circuits of two converters. In the presented case, the coupling was the result of bringing the grounded wires to a close distance of $1 \mathrm{~mm}$. As a result of coupling, the oscillatory signal suppressed from one circuit got through to the other. Therefore, this result highlights the phenomenon of voltage ringing aggregation.

\section{Mathematical ASSUMPTIONS}

On Fig. 4 the difference between the primary and coupled voltage signal is visible. However, a simplifying assumption
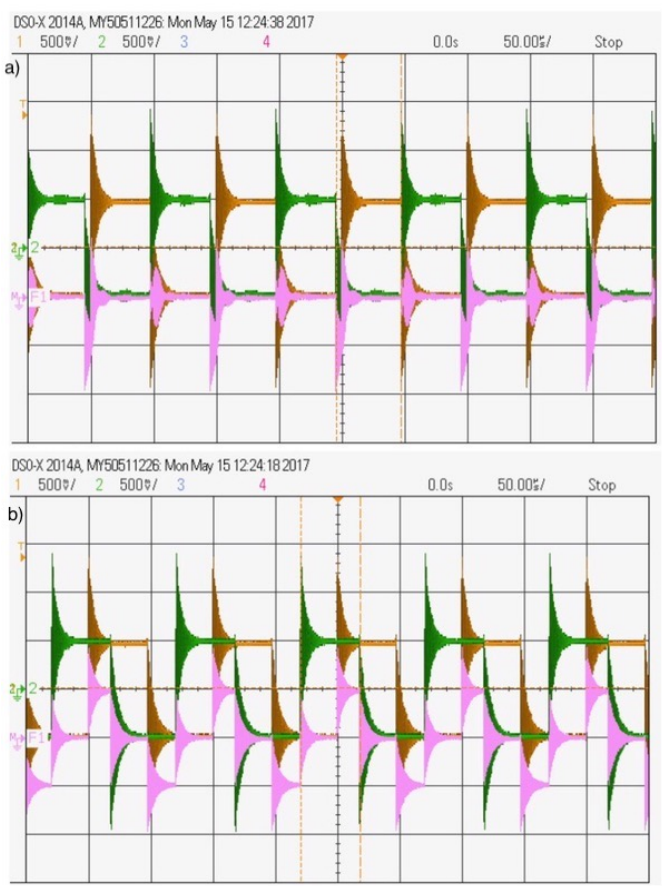

Fig. 3. Damped oscillation voltages of two converters with a) synchronous and b) asynchronous control, measured on the load side.
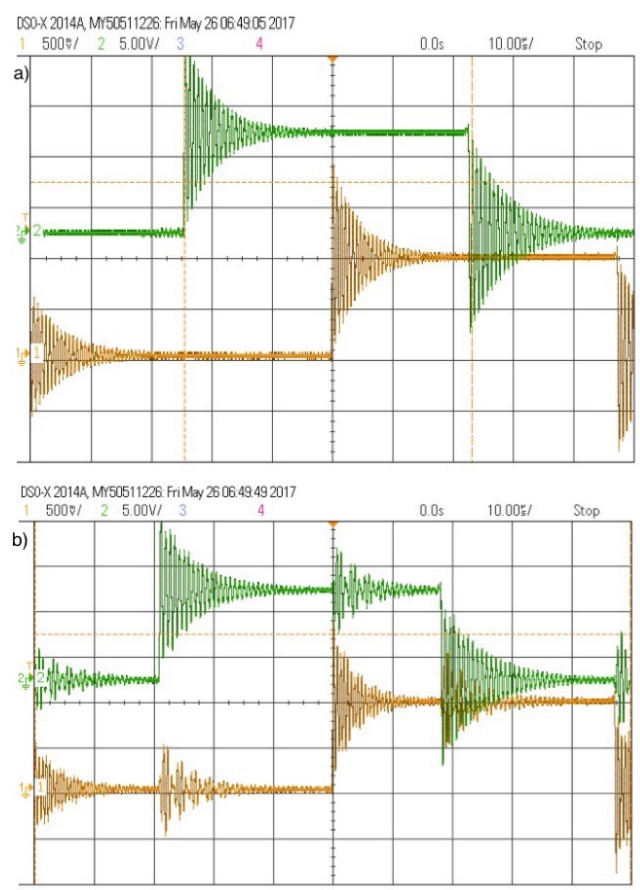

Fig. 4. Voltage of two asynchronously controlled converters: a) without coupling and b) with coupling, measured on the load side.

is that the two are identical. Single oscillatory damped voltage can be expressed using the following relation

$$
v(t)=v_{0} \sin (2 \pi f t) e^{-\lambda t} \mathbf{1}(t),
$$

where: 
- $v_{0}$ is the ringing gain,

- $f$ is the ringing frequency,

- $\lambda$ is the damping factor,

- $\mathbf{1}(t)$ is the Heaviside function.

Let $N$ be the number of converters and let $t_{k, j, O N}$ and $t_{k, j, O F F}$ be, respectively, $k+1$-th switching-on and switchingoff instant of $j$-th converter before time $t$, where $j=1, \ldots, N$. Assuming all converters operate in steady-state mode, the aggregated voltage interference $V(t)$ at time $t$ can be formally expressed as

$$
V(t)=\sum_{j=1}^{N} \sum_{k=0}^{\infty} v\left(t-t_{k, j, O N}\right)-v\left(t-t_{k, j, O F F}\right) .
$$

Given a tolerance $\varepsilon$ the domain of the signal represented by (1) can be limited to such $t$ which satisfy $0 \leq t \leq t_{\varepsilon}$, where

$$
t_{\varepsilon}=\frac{\log v_{0}-\log \varepsilon}{\lambda} .
$$

Notice that for sufficiently large $\lambda$, value of the oscillatory voltage can be deemed as negligibly small after one switching, i.e $|v(t)| \leq \varepsilon$, if $t_{\varepsilon} \leq p_{c}$, where $p_{c}$ is the switching period of the converter. Thus, the value of interference $V(t)$ may be expressed only in the terms of one switching before time $t$. Without loss of generality it can be assumed that the first time instant at which the interference signal is observed is fixed at 0 . Let $t_{j}$ be the last switching-on instant of $j$-th converter before time 0 , for $j=1, \ldots, N$ and let $d$ be the duty cycle of PWM used to control the switching pattern of the converter. Then, switching instants $t_{j, O N}=t_{0, j, O N}$ and $t_{j, O F F}=t_{0, j, O F F}$ of $j$-th converter before time $t$ can be identified by the following formulas

$$
\begin{aligned}
t_{j, \text { ON }} & =\left\lfloor\frac{t-t_{j}}{p_{c}}\right\rfloor p_{c}+t_{j}, \\
t_{j, \text { OFF }} & =\left\lfloor\frac{t-t_{j}}{p_{c}}-d\right\rfloor p_{c}+d p_{c}+t_{j},
\end{aligned}
$$

where $\lfloor\cdot\rfloor$ is the floor function. Therefore, signal $V(t)$ is represented using equation

$$
V(t)=\sum_{j=1}^{N} v\left(t-t_{j, O N}\right)-v\left(t-t_{j, O F F}\right) .
$$

\section{PRoblem Formulation}

Suppose that data transmission is observed, where datachecking moments are fixed and there is a time interval $\Delta t$ between each of the consecutive checking instants. If the absolute value of the interference $V(t)$ at some time $t$ is greater than a certain threshold $V_{t h}>0$, then superposition of the interference and the carrier of data transmission may bring about an increase or decrease in carrier's voltage level, resulting in an unexpected change of data value. An assumption leading to simplification of the transmission scheme is that the time needed for data checking is negligibly small, so that values of the interference are observed in discrete time instants $p \Delta t$, where $p=0,1, \ldots, K-1$ and $K$ is the length of transmission, e.g. number of bits. This situation is depicted on Fig. 5. The

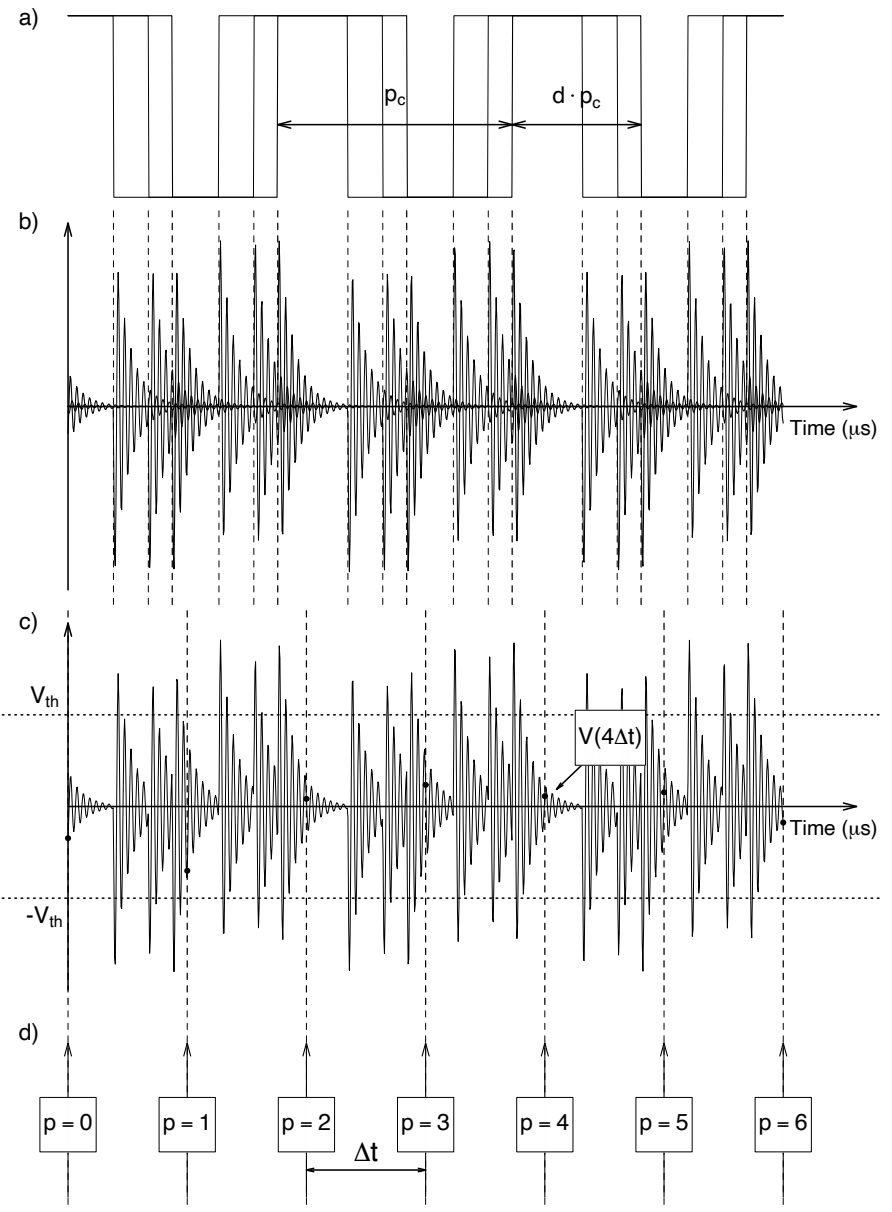

Fig. 5. The visual explanation of a problem: a) carrier wave of 3 PWM operated DC/DC converters in steady-state mode, b) the damped oscillation voltages fired by the switching action of the converters, c) the interference pattern as a sum of interferences caused by each of the converters, d) bit checking instants $p \Delta t$.

following questions arise:

1) What is the value of the interference $V(p \Delta t)$ at datachecking moments?

2) What is the probability that data will be corrupted?

3) What is the relationship between the number of converters, switching frequency and errors on the communication system?

In the next section a simple approach to consider these questions is presented.

\section{SimUlation APPROACH}

This section briefly discusses the approach taken to quantify errors induced on a system by operation of DC/DC converters. It should be noted that the following is a first step of the solution and a fully validated error model will be developed in the future.

\section{A. Monte Carlo simulation}

The plot on the top on Fig. 6 shows the interference voltage represented by (3). Notice, that a repeatable pattern of 
interference voltage occurring after every switching is visible. Therefore, only the first time period $\left\langle 0, p_{c}\right\rangle$ is taken into consideration and Monte Carlo simulations with appropriately chosen sampling rate are conducted to obtain values of the interference $V(t)$. It is assumed that $t_{j}$ for every $j=1, \ldots, N$ follows a continuous uniform distribution $\mathcal{U}\left(-p_{c}, 0\right)$.

\section{B. Distribution of times between interferences}

The plot on the bottom of Fig. 6 presents an example of estimation of the times between interferences. The absolute value of the voltage $V(t)$ is compared with the threshold $V_{t h}$ and the times between successive blocks of interference exceeding the threshold are collected. Let $d t$ be a random variable describing the time between interferences and $d t_{1}, d t_{2}, \ldots, d t_{n}$ a sequence of its realizations, as presented on Fig. 6, where $n=3$. Voltage values obtained via MC simulations are used to find the distribution of $d t$. The parameters of this distribution have to be estimated so that a complete error model can be developed.

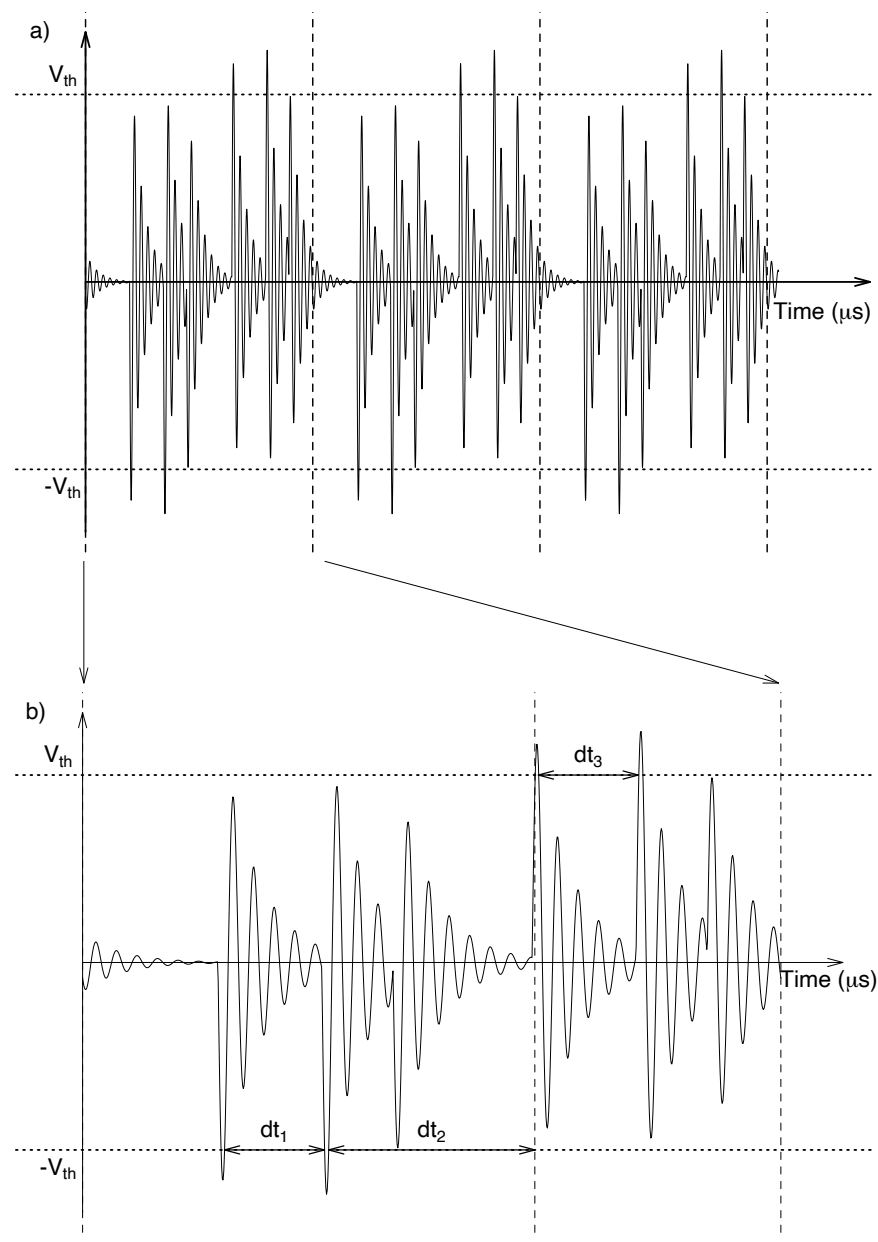

Fig. 6. Repetitive pattern of aggregated interference on panel a) and the realization of times between interferences $d t_{1}, d t_{2}, d t_{3}$ on panel b).

\section{PRELiminary Results}

Preliminary results using the above described simulation approach were collected with the following parameters:
- $v_{0}=6 \mathrm{~V}$,

- $f=5.5 \mathrm{MHz}$

- $\lambda=10^{6}$,

- $N=2, \ldots, 10$,

- $d=0.5$,

- minimum switching frequency $f_{c}=1 / p_{c}=20 \mathrm{kHz}$,

- maximum switching frequency $f_{c}=1 / p_{c}=200 \mathrm{kHz}$,

- sampling frequency $1.408 \mathrm{GHz}$.

Fig. 7 shows the results of the simulation. Times between interferences $d t$ are shown on base 10 logarithmic scale for clarity of the plots. In an idealized, theoretical situation where the communication is continuous, times between interferences would indicate periods between disruptions. Even though such a situation is not encountered in real life, some conclusions can be drawn based on the intuition behind this approach. Fig. 7 a) presents a distribution of $d t$ with $N=5$ converters and switching frequency $f_{c}=20 \mathrm{kHz}$. As it can be seen, the distribution is left-skewed and characterize with long left tail, which means that there is a small but nonzero probability of obtaining small values of $d t$. The highest probability is obtained when $d t$ is between $1 \mu \mathrm{s}$ and $10 \mu \mathrm{s}$, which in continuous case would indicate that most of the disruptions would be happening on that scale. There is a smaller mode between $0.01 \mu \mathrm{s}$ and $0.1 \mu \mathrm{s}$, which emerges even more when the switching frequency is increased, as shown on Fig. 7 c). There is a clear relationship between the mean value and number of converters or switching frequency, i.e. the mean value decreases hyperbolically with increasing number of converters or with increasing switching frequency. This relationship, indicated by a smooth line on Fig. $7 \mathrm{~b}$ ), testifies that in a system with more converters present or with higher switching frequency, times between disruptions would be on average shorter.

\section{DISCUSSION}

The simulation results are taken with the assumption that times between interferences are described by continuous probability distributions. However, the true impact of the interferences on the communication channel has to take into account that time checking instants are discrete and the switching frequency $f_{c}$ may differ from the data transmission frequency $f_{d}$. In the paper [13] the model of error probability caused by operation of a single PWM controlled DC/DC converter was presented and a relationship between the probability of errors and the period between communication checking instants $\Delta t$ and switching period $p_{c}$ was shown. Intuitively, if $\Delta t$ is a multiple of $p_{c}$ (or the other way around), then errors caused by interference described in terms of damped oscillations, can be deterministic. However, in real situations it is not possible to have a full control over both the communication channel and the operation of $\mathrm{dc} / \mathrm{dc}$ converters, therefore a statistical approach is needed to quantify and describe situations in which errors can be induced on a system. Fig. 8 presents the results of simulation of damped harmonic oscillation signal interfering with communication channel. Data transmission frequency $f_{d}$ was set to $17520 \mathrm{~Hz}$ and the values of interference at data-checking moments $V(p \Delta t)$ were collected for 
a)

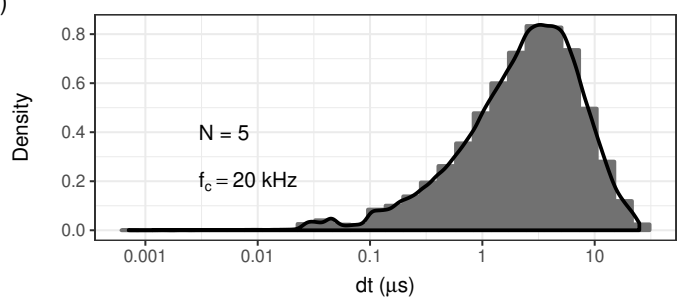

b)

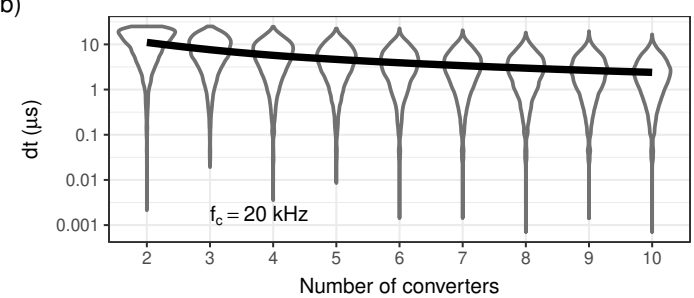

c)

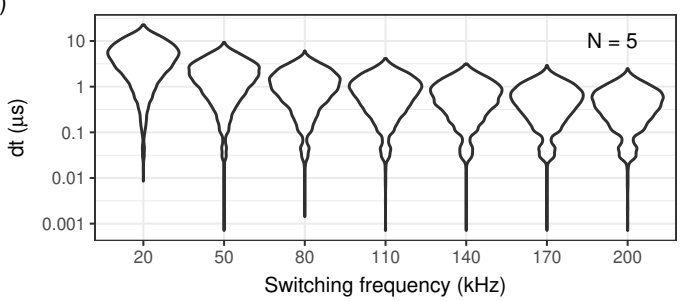

Fig. 7. Preliminary results of the simulations: a) an example of the density of $d t$ with $N=5$ and $f_{c}=20 \mathrm{kHz}, \mathrm{b}$ ) violin plots highlighting the relationship between the density of $d t$ and number of converters at $f_{c}=20 \mathrm{kHz}, \mathrm{c}$ ) violin plots showing the relationship between the density of $d t$ and switching frequency $f_{c}$.

$p=0,1, \ldots, 1000$ and checked for exceeding the threshold $V_{t h}=3 \mathrm{~V}$. The exceedance rate was then calculated as the number of values exceeding the threshold over number of checked values. The general conclusion is that increased switching frequency allows for generating higher number of errors, which is in line with the conclusion drawn from Monte Carlo simulations presented in Section VI. Furthermore, the number of possible values for the exceedance rate increases with increasing switching frequency. However, at low switching frequencies, multi modal densities are present, which suggest that a relationship between switching frequency, data transmission frequency and the exceedance rate cannot be characterized using standard unimodal distributions. Thus, the interesting aspect of this research is extending the deterministic model presented in [13] over systems with multiple converters.

\section{CONCLUSIONS}

In this paper, a statistical approach to quantify time-domain impact of common mode voltage induced on a communication system by the operation of several sources of emissions was presented. To simplify the reasoning, deterministic PWM operated DC/DC converters were chosen as emission sources. A mathematical formulation of time-domain impact

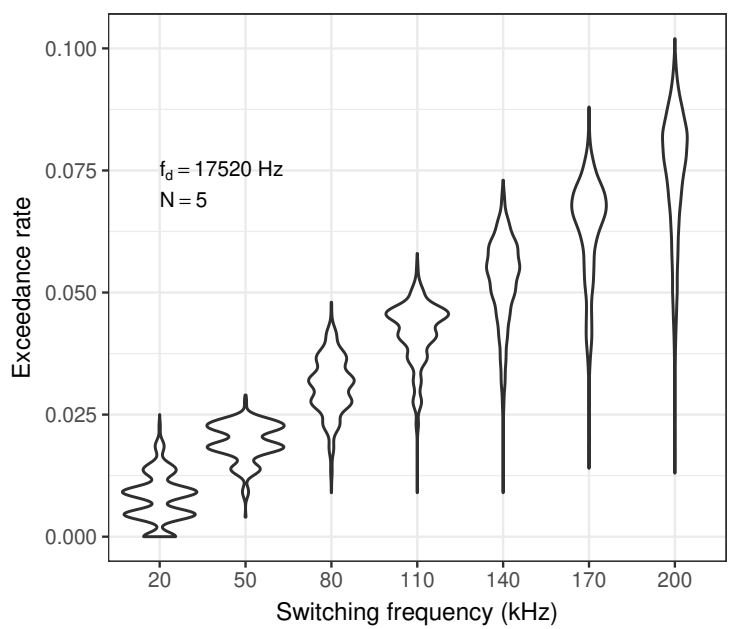

Fig. 8. Violin plots of threshold exceedance rates, assuming data transmission frequency is equal to $17520 \mathrm{~Hz}$ and the number of converters is 5

of interference affecting communication link was derived and simulation approach allowing to obtain distribution of times between interferences using a simplified interference model was proposed. Results of the preliminary simulations suggest that in systems with more than 2 converters, times between interferences are on average shorter than in those with only 2 converters, and that the relationship between times between interferences and number of converters is nonlinear and inversely proportional to the number of converters. Similar relationship was observed with fixed number of converters and increasing switching frequency, where the inverse proportion was with respect to the switching frequency. In both cases the distributions of times between interferences after logarithmic transformation were left-skewed and characterized with long left tails, suggesting small number of short times between interferences, even on the scale nanoseconds. Preliminary simulations of a discrete communication system with fixed data transmission frequency confirm that the average number of possible disruptions caused by the operation of several converters is increasing alongside with the switching frequency. However, the uncertainty about the number of disruptions is also increasing, which is observed by the variance expansion. Therefore, further work is needed to capture the relationship between the rate of disruptions, number of converters and switching frequency. In future work, the results of numerical simulations will be verified by experimental measurements and the mathematical model will be revised and further developed.

\section{REFERENCES}

[1] R. Smolenski, Conducted electromagnetic interference (EMI) in smart grids, vol. 68 of Power Systems. Springer London, 012012.

2] Y. Koyama, M. Tanaka, and H. Akagi, "Modeling and analysis for simulation of common-mode noises produced by an inverter-driven air conditioner," IEEE Transactions on Industry Applications, vol. 47, pp. 2166-2174, Sep. 2011.

[3] L. Ran, S. Gokani, J. Clare, K. J. Bradley, and C. Christopoulos, "Conducted electromagnetic emissions in induction motor drive systems. i. time domain analysis and identification of dominant modes," IEEE Transactions on Power Electronics, vol. 13, pp. 757-767, July 1998. 
[4] H. Bishnoi, A. C. Baisden, P. Mattavelli, and D. Boroyevich, "Analysis of emi terminal modeling of switched power converters," IEEE Transactions on Power Electronics, vol. 27, pp. 3924-3933, Sep. 2012.

[5] B. t. Have, T. Hartman, N. Moonen, and F. Leferink, "Why frequency domain tests like iec 61000-4-19 are not valid; a call for time domain testing," in 2019 International Symposium on Electromagnetic Compatibility - EMC EUROPE, pp. 124-128, Sep. 2019.

[6] R. Smolenski, J. Bojarski, A. Kempski, and P. Lezynski, "Time-domainbased assessment of data transmission error probability in smart grids with electromagnetic interference," IEEE Transactions on Industrial Electronics, vol. 61, pp. 1882-1890, April 2014.

[7] N. Ginot, M. A. Mannah, C. Batard, and M. Machmoum, "Application of power line communication for data transmission over pwm network," IEEE Transactions on Smart Grid, vol. 1, pp. 178-185, Sep. 2010.

[8] B. J. A. M. van Leersum, R. B. Timens, F. J. K. Buesink, and F. B. J. Leferink, "Time domain methods for the analysis of conducted interference on the power supply network of complex installations," in 2014 International Symposium on Electromagnetic Compatibility, pp. 605-610, Sep. 2014.

[9] J. Bojarski, R. Smolenski, A. Kempski, and P. Lezynski, "Pearson's random walk approach to evaluating interference generated by a group of converters," Applied Mathematics and Computation, vol. 219, no. 12, pp. $6437-6444,2013$

[10] P. M. Ivry, O. A. Oke, D. W. P. Thomas, and M. Sumner, "Predicting conducted emissions of multiple vscs using dimension reduction technique," in 2015 IEEE International Symposium on Electromagnetic Compatibility (EMC), pp. 213-218, Aug 2015.

[11] M. L. Heldwein, J. Biela, H. Ertl, T. Nussbaumer, and J. W. Kolar, "Novel three-phase $\mathrm{cm} / \mathrm{dm}$ conducted emission separator," IEEE Transactions on Industrial Electronics, vol. 56, pp. 3693-3703, Sep. 2009.

[12] T. T. Nguyen, N. Nguyen, and N. R. Prasad, "Eliminated commonmode voltage pulsewidth modulation to reduce output current ripple for multilevel inverters," IEEE Transactions on Power Electronics, vol. 31, pp. 5952-5966, Aug 2016

[13] R. Smoleński, J. Bojarski, P. Lezynski, and Z. Sadowski, "Diophantine equation based model of data transmission errors caused by interference generated by dc-dc converters with deterministic modulation," Bulletin of the Polish Academy of Sciences: Technical Sciences, vol. 64, no. No 3, pp. 575-580, 2016. 